\title{
Pseudo-Hermitian approach to Goldstone's theorem in non-Abelian non-Hermitian quantum field theories
}

\author{
Andreas Fring $\odot^{*}$ and Takanobu Taira $\odot^{\dagger}$ \\ Department of Mathematics, City, University of London, Northampton Square, \\ London EC1V OHB, United Kingdom
}

(Received 11 November 2019; accepted 28 January 2020; published 19 February 2020)

\begin{abstract}
We generalize previous studies on the extension of Goldstone's theorem from Hermitian to nonHermitian quantum field theories with Abelian symmetries to theories possessing a glocal non-Abelian symmetry. We present a detailed analysis for a non-Hermitian field theory with two complex two component scalar fields possessing an $S U(2)$ symmetry and indicate how our findings extend to the general case. In the PT-symmetric regime (parity and time-reversal) and at the standard exceptional point the Goldstone theorem is shown to apply, although different identification procedures need to be employed. At the zero exceptional points the Goldstone boson can not be identified. Comparing our approach, based on the pseudo-Hermiticity of the model, to an alternative approach that utilizes surface terms to achieve compatibility for the non-Hermitian system, we find that the explicit forms of the Goldstone boson fields are different.
\end{abstract}

DOI: 10.1103/PhysRevD.101.045014

\section{INTRODUCTION}

The extension from quantum field theories with Hermitian actions to those with non-Hermitian actions has been addressed recently for various concrete systems, such as scalar field theory with imaginary cubic selfinteraction terms [1,2], field theoretical analogs to the deformed harmonic oscillator [3], non-Hermitian versions with a field theoretic Yukawa interaction [4-7], free fermion theory with a $\gamma_{5}$-mass term and the massive Thirring model [8], $\mathcal{P} \mathcal{T}$-symmetric versions of quantum electrodynamics $[9,10]$, and $\mathcal{P} \mathcal{T}$-symmetric quantum field theories in higher dimensions [11].

The generalizations also include Goldstone's theorem $[12,13]$ and the Higgs mechanism [14-20]. Both of these mechanisms are governed by the continuous symmetries of the theories, global or local, respectively, that might by spontaneously broken by some vacuum states. The special feature of non-Hermitian systems is that an additional discrete antilinear symmetry [21] is superimposed on top of the continuous symmetries, that can also be spontaneously broken, albeit not exclusively for the ground state in this case. The regime in which the discrete symmetry is broken

\footnotetext{
a.fring@city.ac.uk

takanobu.taira@city.ac.uk
}

Published by the American Physical Society under the terms of the Creative Commons Attribution 4.0 International license. Further distribution of this work must maintain attribution to the author(s) and the published article's title, journal citation, and DOI. Funded by SCOAP . is regarded as unphysical. In general, the antilinear symmetry separates the parameter space of the theory into regimes of different types of behavior. The physical subspace is bounded by the values for which the eigenvalues of the mass squared matrix acquire an exceptional point, a singularity or becomes zero. It is the interplay between these two types of symmetries, continuous and discrete, that produce very interesting and novel behavior when compared to the standard Hermitian setting.

There is a well-known problem that seems to suggest that non-Hermitian quantum field theories are inconsistent, see e.g., $[8,22,23]$. However, just as for non-Hermitian quantum theories [24-27] there are methods and techniques to overcome these issues to obtain a perfectly consistent theory. The conundrum for the quantum fields theories consists of the feature that the two sets of equations of motion, derived from functionally varying the action with respect to the scalar fields on one hand and with respect to their complex conjugates on the other, are incompatible. So far two distinct alternative propositions have been made to overcome this issue. Alexandre, Ellis, Millington, and Seynaeve proposed to apply a nonstandard variational principle by keeping some nonvanishing surface terms [17,20] or, in line with the pseudo-Hermitian $/ \mathcal{P} \mathcal{T}$-symmetric quantum mechanical approach [24-27], one may seek a consistent equivalent similarity transformed Hermitian action, as pursued by Mannheim and the present authors [18,19]. While some features are the same in both approaches, e.g., both versions predict the same number of massless Goldstone bosons that is expected from Goldstone's theorem, they also differ in several aspects. While in the former proposition Noether's 
theorem is evaded the latter is based on the standard variational principle leading to standard Noether currents. Moreover with regard to the Higgs mechanism the "surface term approach" predicts that the gauge particle becomes massive in the local case [20], whereas the "pseudoHermitian approach" leads to a theory in which the gauge particle remains massless at the exceptional point [18]. Here we also find that the explicit form of the Goldstone bosons differs.

Previous considerations were focused on the analysis of non-Hermitian systems with a global and local Abelian $U(1)$ symmetry, they were recently extended to nonAbelian theories within the surface term approach [20]. Here we also extend these studies to the non-Abelian case by applying the pseudo-Hermitian approach. We analyze in detail a non-Hermitian scalar field theory with two complex two component scalar fields possessing an $S U(2)$ symmetry and an overall discrete antilinear symmetry. We compare our results to those obtained in [20] by means of the surface term approach.

In Sec. II we discuss the generalities of the pseudoHermitian approach to achieve compatibility in nonHermitian quantum field theories, with an emphasis on how it modifies the identification of the mass squared matrix and Goldstone's theorem. In Sec. III we discuss a concrete model with two complex scalar fields in the fundamental representation, by deriving an equivalent Hermitian action for the model, discussing its $S U(2)$ symmetry, its vacua, mass squared matrices, physical regions, and identifications of the Goldstone bosons in the different regimes. We state our conclusions in Sec. IV.

\section{PSEUDO-HERMITIAN APPROACH TO SPONTANEOUSLY BROKEN SYMMETRIES}

We consider here complex scalar quantum field theories described by actions of the following generic type:

$$
\mathcal{I}=\int d^{4} x\left[\partial_{\mu} \phi \partial^{\mu} \phi^{*}-V(\phi)\right],
$$

with $n$-component complex scalar fields $\phi=\left(\phi_{1}, \ldots, \phi_{n}\right)$ and potential $V(\phi)$. The action is assumed to possess three general properties: (i) It is invariant under a global continuous symmetry $\phi \rightarrow \phi+\delta \phi$ with $V(\phi)=$ $V(\phi+\delta \phi)$. The symmetry is, for instance, generated by a Lie group $\mathbf{g}$ with Lie algebraic generators $T$, so that being global implies an infinitesimal change $\delta \phi=\alpha T \phi$ with $\alpha$ being a small parameter and $\partial_{\mu}(\alpha T)=0$. (ii) It is invariant under a discrete antilinear symmetry $\phi\left(x_{\mu}\right) \rightarrow$ $U \phi^{*}\left(-x_{\mu}\right)$, with $U$ being a constant unitary matrix. These symmetries may be viewed as modified $\mathcal{C P} \mathcal{T}$ symmetries. When $U \rightarrow \mathbb{I}$ the symmetry reduces to the standard $\mathcal{C P} \mathcal{T}$ symmetry. (iii) The potential $V(\phi)$ is not Hermitian, that is $V(\phi) \neq V^{\dagger}(\phi)$.
At first sight such type of theories appears to be inconsistent as the two sets of equations of motion obtained by functionally varying the action $\mathcal{I}$ separately with respect to the fields $\phi_{i}$ and $\phi_{i}^{*}, \delta \mathcal{I}_{n} / \delta \phi_{i}=0$ and $\delta \mathcal{I}_{n} / \delta \phi_{i}^{*}=0$ are in general incompatible when $U \neq \mathbb{I}$. One may, however, overcome this problem by using a nonstandard variational principle combined with keeping some nonvanishing surface terms $[17,20]$ or alternatively by exploiting the fact that the content of the theory is unaltered as long as the equal time commutation relations are preserved and carry out a similarity transformation that guarantees that feature $[8,18,19]$. Hence, in the latter approach one seeks a Dyson map $\eta$, named this way in analogy to its quantum mechanical counterpart [28], to construct a new equivalent action

$$
\hat{\mathcal{I}}=\eta \mathcal{I} \eta^{-1}=\int d^{4} x\left[\partial_{\mu} \phi \hat{I} \partial^{\mu} \phi^{*}-\hat{V}(\phi)\right],
$$

with the difference that now the transformed potential is Hermitian, i.e., it remains invariant under complex conjugation $\hat{V}(\phi)=\hat{V}^{\dagger}(\phi)$. The matrix $\hat{I}$ is a result of the similarity transformation.

Next it is in general useful to convert the complex scalar field theory into one involving only real valued fields by decomposing the $n$ complex scalar fields into real and imaginary parts as $\phi=1 / \sqrt{2}(\varphi+i \chi)$ with $\varphi, \chi \in \mathbb{R}$. Defining then a real $2 n$-component field $\Phi=\left(\varphi_{1}, \ldots, \varphi_{n}, \chi_{1}, \ldots, \chi_{n}\right)$, possibly with the fields in different order to block diagonalize the mass squared matrix, the new action $\hat{\mathcal{I}}$ may be rewritten as

$$
\hat{\mathcal{I}}=\int d^{4} x\left[\frac{1}{2} \partial_{\mu} \Phi I \partial^{\mu} \Phi^{*}-\hat{V}(\Phi)\right] .
$$

Analyzing the action in this form, the extension of Goldstone's theorem from the Hermitian to the nonHermitian case is easily established. At first we identify various types of vacua $\Phi_{0}$ by solving

$$
\left.\frac{\partial \hat{V}(\Phi)}{\partial \Phi}\right|_{\Phi=\Phi_{0}}=0 .
$$

The continuous global symmetry $\Phi \rightarrow \Phi+\delta \Phi$, i.e., $\hat{V}(\Phi)=\hat{V}(\Phi+\delta \Phi)=\hat{V}(\Phi)+\nabla \hat{V}(\Phi)^{T} \delta \Phi$, then implies

$$
\frac{\partial \hat{V}(\Phi)}{\partial \Phi_{i}} \delta \Phi_{i}(\Phi)=0
$$

Differentiating this equation with respect to $\Phi_{j}$ and evaluating the result at a vacuum $\Phi_{0}$, determined by (2.4), yields

$\left.\frac{\partial^{2} \hat{V}(\Phi)}{\partial \Phi_{j} \partial \Phi_{i}}\right|_{\Phi=\Phi_{0}} \delta \Phi_{i}\left(\Phi_{0}\right)+\left.\left.\frac{\partial \hat{V}(\Phi)}{\partial \Phi_{i}}\right|_{\Phi=\Phi_{0}} \frac{\partial \delta \Phi_{i}(\Phi)}{\partial \Phi_{j}}\right|_{\Phi=\Phi_{0}}=0$. 
Since the last term vanishes, due to (2.4), we are left with two options to solve (2.6). Either the vacuum is left invariant such that $\delta \Phi_{i}\left(\Phi_{0}\right)=0$ or the vacuum breaks the global symmetry and $\delta \Phi_{i}\left(\Phi_{0}\right) \neq 0$. Denoting $\theta_{0}:=$ $\delta \Phi_{i}\left(\Phi_{0}\right)$ and multiplying (2.6) by $\hat{I}$ we obtain

$$
\hat{I} H\left(\Phi_{0}\right) \theta_{0}=M^{2} \theta_{0}=0,
$$

where $H\left(\Phi_{0}\right)$ is the Hessian of the potential $\hat{V}(\Phi)$ evaluated at the vacuum $\Phi_{0}$ and $M^{2}$ is the mass squared matrix. The occurrence of the matrix $\hat{I}$ results from the similarity transformation and is therefore the trace of the feature that the potential is non-Hermitian. It also has the effect that $M^{2}$ is no longer Hermitian either. We can now read off Goldstone's theorem for non-Hermitian systems from (2.7). When the vacuum is left invariant by the global symmetry transformation we have $\theta_{0}=0$ so that there is no restriction on $M^{2}$. However, when the vacuum breaks the global symmetry we have $\theta_{0} \neq 0$ so that $\theta_{0}$ becomes a null vector for $M^{2}$. Thus, in this case we have a zero mass particle, that is identified as a Goldstone boson.

Assuming that the symmetry is generated by a Lie group g, we may repeat this argument for each Lie algebraic generator $T$ so that we obtain a Goldstone boson for each generator that when acting on the vacuum $\Phi_{0}$ produces a different one. The crucial difference, when compared to the scenario with Hermitian potentials, is that here $M^{2}$ is also not Hermitian. This means that the physical regimes are determined by the discrete antilinear symmetries. Referring to this symmetry as $\mathcal{P} \mathcal{T}$-symmetry [25,27] in a wider sense, we may encounter $\mathcal{P} \mathcal{T}$-symmetric regimes with real mass spectra, exceptional points with nondiagonalizable mass matrix, zero exceptional points, singularities, and a spontaneously broken $\mathcal{P} \mathcal{T}$-symmetric regime with unphysical complex conjugate masses. Similar as in [19] we distinguish here between a standard exceptional point where two eigenvalues coalesce and become complex thereafter and a zero exceptional point at which one positive real eigenvalue coincides with a zero eigenvalue and remains real thereafter. As shown in [19] the identification of the Goldstone boson is different in these regimes and in parts impossible.

Below we will also make use of the general property that the expansions around two vacua, say $\phi_{0}^{1}$ and $\phi_{0}^{2}$, that are related by the symmetry transformation $\mathcal{T}$ of the potential $V(\phi)=V(\mathcal{T} \phi)$ as $\mathcal{T} \phi_{0}^{1}=\phi_{0}^{2}$ with $\mathcal{T}^{T}=\mathcal{T}^{-1}$ yield to theories with mass squared matrix possessing the same eigenvalues. This can be seen from

$$
\begin{aligned}
V\left(\phi+\phi_{0}^{1}\right) & =V\left(\phi+\mathcal{T}^{-1} \phi_{0}^{2}\right)=V\left(\mathcal{T}^{-1}\left(\mathcal{T} \phi+\phi_{0}^{2}\right)\right)=V\left(\mathcal{T} \phi+\phi_{0}^{2}\right) \\
& =V\left(\phi_{0}^{2}\right)+\frac{1}{2} \phi^{T} \mathcal{T}^{T} H\left(\phi_{0}^{2}\right) \mathcal{T} \phi+\cdots=V\left(\phi_{0}^{2}\right)+\frac{1}{2} \phi^{T} H\left(\phi_{0}^{2}\right) \phi+\cdots \\
& =V\left(\phi+\phi_{0}^{2}\right) .
\end{aligned}
$$

As the kinetic term is invariant by itself no modification of the mass squared matrix will arise from there, apart form the multiplication by $\hat{I}$ as a result of the non-Hermitian nature. Thus we may employ the symmetry to transform the vacuum into the most convenient form for analysis without altering the physics, such as the eigenvalue spectrum of the mass matrix.

\section{A $\mathcal{C} \mathcal{P} \mathcal{T}$-SYMMETRIC NON-HERMITIAN MODEL WITH GLOBAL SU(2) SYMMETRY}

Let us now verify the previous general statements for a more concrete system. We consider the action

$$
\mathcal{I}_{s u 2}=\int d^{4} x\left[\sum_{i=1}^{2}\left(\left|\partial_{\mu} \phi_{i}\right|^{2}+m_{i}^{2}\left|\phi_{i}\right|^{2}\right)-\mu^{2}\left(\phi_{1}^{\dagger} \phi_{2}-\phi_{2}^{\dagger} \phi_{1}\right)-\frac{g}{4}\left|\phi_{1}\right|^{4}\right],
$$

where the two complex scalar fields $\phi_{i}=\left(\phi_{i}^{1}, \phi_{i}^{2}\right)^{T}$, $i=1,2$, are taken to be in the fundamental or spin $1 / 2$ representation of $S U(2)$ and $g, \mu \in \mathbb{R}$ are constants. We allow here for $m_{i} \in \mathbb{R}$ or $m_{i} \in i \mathbb{R}$, so that $m_{i} \rightarrow c_{i} m_{i}$ with $c_{i}=1$ or $c_{i}=-1$, respectively, takes care of these two possibilities. For simplicity we suppress the parameters $c_{i}$ until we analyze the physical parameter space in Sec. III E. We observe that the action $\mathcal{I}_{s u 2}$ has the aforementioned three properties. It is invariant under a global continuous symmetry $\phi_{j}^{k} \rightarrow \phi_{j}^{k}+\delta \phi_{j}^{k} \quad$ where $\delta \phi_{j}^{k}=i \alpha_{a} T_{a}^{k l} \phi_{j}^{l}$ with $S U(2)$-Lie algebraic generators $T_{a}$, is invariant under two discrete antilinear symmetries $\mathcal{C P} \mathcal{T}_{ \pm}: \phi\left(x_{\mu}\right) \rightarrow \pm \sigma_{3} \phi^{*}\left(-x_{\mu}\right)$, with $\sigma_{3}$ denoting one of the Pauli spin matrices, and the potential $V(\phi)$ in (3.1) is evidently not Hermitian. We note that in the surface term approach [20] the antilinear symmetries are implemented differently by $\mathcal{P} \mathcal{T}$ not acting on the arguments of the fields. 


\section{A. Equivalent Hermitian actions}

More explicitly in components and transformed to the real fields $\varphi_{j}^{k}, \chi_{j}^{k} \in \mathbb{R}$, via $\phi_{j}^{k}=1 / \sqrt{2}\left(\varphi_{j}^{k}+i \chi_{j}^{k}\right)$, the action $\mathcal{I}_{s u 2}$ reads

$$
\begin{aligned}
\mathcal{I}_{s u 2}= & \int d^{4} x\left[\frac{1}{2} \sum_{j, k=1}^{2}\left(\partial_{\mu} \varphi_{j}^{k}\right)^{2}+\left(\partial_{\mu} \chi_{j}^{k}\right)^{2}+m_{j}^{2}\left(\varphi_{j}^{k}\right)^{2}+m_{j}^{2}\left(\chi_{j}^{k}\right)^{2}+i 2 \mu^{2}\left(\chi_{1}^{k} \varphi_{2}^{k}-\varphi_{1}^{k} \chi_{2}^{k}\right)\right. \\
& \left.-\frac{g}{16}\left[\left(\varphi_{1}^{1}\right)^{2}+\left(\varphi_{1}^{2}\right)^{2}+\left(\chi_{1}^{1}\right)^{2}+\left(\chi_{1}^{2}\right)^{2}\right]^{2}\right]
\end{aligned}
$$

As indicated above, the direct functional variation of this action will lead to inconsistent equations of motion and we therefore seek a suitable similarity transformation to resolve this issue. Using the Dyson map

$$
\eta=e^{\frac{\pi}{2} \int d^{3} x \Pi_{2}^{\varphi, 1}(\mathbf{x}, t) \varphi_{2}^{1}(\mathbf{x}, t)} e^{\frac{\pi}{2} \int d^{3} x \Pi_{2}^{\varphi, 2}(\mathbf{x}, t) \varphi_{2}^{2}(\mathbf{x}, t)} e^{\frac{\pi}{2} \int d^{3} x \Pi_{2}^{\chi, 1}(\mathbf{x}, t) \chi_{2}^{1}(\mathbf{x}, t)} e^{\frac{\pi}{2} \int d^{3} x \Pi_{2}^{x_{2}}(\mathbf{x}, t) \chi_{2}^{2}(\mathbf{x}, t)},
$$

with canonical momenta $\Pi_{j}^{\varphi, k}=\partial_{t} \varphi_{j}^{k}, \Pi_{j}^{\chi, k}=\partial_{t} \chi_{j}^{k}$ and $\Pi_{j}^{\phi, k}=\partial_{t} \phi_{j}^{k}, j, k=1,2$, the adjoint actions of $\eta$ on the real and complex scalar fields and canonical momenta is computed to

$$
\begin{array}{lll}
\eta \varphi_{j}^{k} \eta^{-1}=(-i)^{\delta_{2 j}} \varphi_{j}^{k}, & \eta \chi_{j}^{k} \eta^{-1}=(-i)^{\delta_{2 j}} \chi_{j}^{k}, & \eta \phi_{j}^{k} \eta^{-1}=(-i)^{\delta_{2 j}} \phi_{j}^{k}, \\
\eta \Pi_{j}^{\varphi, k} \eta^{-1}=i^{\delta_{2 j}} \Pi_{j}^{\varphi, k}, & \eta \Pi_{j}^{\chi, k} \eta^{-1}=i^{\delta_{2 j}} \Pi_{j}^{\chi, k}, & \eta \Pi_{j}^{\phi, k} \eta^{-1}=i^{\delta_{2 j}} \Pi_{j}^{\phi, k}
\end{array}
$$

Thus we can utilize $\eta$ to transform $\mathcal{I}_{s u 2}$ into a Hermitian action, i.e., remaining invariant under complex conjugation,

$$
\begin{aligned}
\hat{\mathcal{I}}_{s u 2}= & \eta \mathcal{I}_{s u 2} \eta^{-1}=\int d^{4} x\left[\sum_{j, k=1}^{2}(-1)^{\delta_{2 j}} \frac{1}{2}\left[\left(\partial_{\mu} \varphi_{j}^{k}\right)^{2}+\left(\partial_{\mu} \chi_{j}^{k}\right)^{2}+m_{j}^{2}\left(\varphi_{j}^{k}\right)^{2}+m_{j}^{2}\left(\chi_{j}^{k}\right)^{2}\right]\right. \\
& \left.+\mu^{2}\left(\chi_{1}^{k} \varphi_{2}^{k}-\varphi_{1}^{k} \chi_{2}^{k}\right)-\frac{g}{16}\left[\left(\varphi_{1}^{1}\right)^{2}+\left(\varphi_{1}^{2}\right)^{2}+\left(\chi_{1}^{1}\right)^{2}+\left(\chi_{1}^{2}\right)^{2}\right]^{2}\right] .
\end{aligned}
$$

It is useful to note here for our analysis and especially with regard to the generalizations to systems with symmetries of higher rank than the action $\hat{\mathcal{I}}_{s u 2}$ can also be cast into a more compact form as

$$
\begin{aligned}
\hat{\mathcal{I}}_{\text {su } 2}=\int d^{4} x \sum_{i=1}^{2} \partial_{\mu} \Phi_{i} I \partial^{\mu} \Phi_{i}+\partial_{\mu} \Psi_{i} I \partial^{\mu} \Psi_{i}+\frac{1}{2} \Phi_{i}^{T} H_{+} \Phi_{i}+\frac{1}{2} \Psi_{i}^{T} H_{-} \Psi_{i} \\
-\frac{g}{16}\left(\Phi_{i}^{T} E \Phi_{i}+\Psi_{i}^{T} E \Psi_{i}\right)^{2}, \\
=\int d^{4} x\left[\partial_{\mu} F \hat{I} \partial^{\mu} F+\frac{1}{2} F^{T} \hat{H} F-\frac{g}{16}\left(F^{T} \hat{E} F\right)^{2}\right],
\end{aligned}
$$

where we defined the matrices and vectors

$$
H_{ \pm}=\left(\begin{array}{cc}
m_{1}^{2} & \pm \mu^{2} \\
\pm \mu^{2} & m_{2}^{2}
\end{array}\right), \quad I=\left(\begin{array}{cc}
1 & 0 \\
0 & -1
\end{array}\right), \quad E=\left(\begin{array}{ll}
1 & 0 \\
0 & 0
\end{array}\right), \quad \Phi_{j}=\left(\begin{array}{c}
\varphi_{1}^{j} \\
\chi_{2}^{j}
\end{array}\right), \quad \Psi_{j}=\left(\begin{array}{c}
\chi_{1}^{j} \\
\varphi_{2}^{j}
\end{array}\right),
$$

$\Phi=\left(\Phi_{1}, \Phi_{2}\right), \quad \Psi=\left(\Psi_{1}, \Psi_{2}\right), \quad F=(\Phi, \Psi)=\left(\varphi_{1}^{1}, \chi_{2}^{1}, \varphi_{1}^{2}, \chi_{2}^{2}, \chi_{1}^{1}, \varphi_{2}^{1}, \chi_{1}^{2}, \varphi_{2}^{2}\right), \quad \operatorname{diag} \hat{I}=\{I, I, I, I\}, \quad \operatorname{diag} \hat{H}=\left\{H_{+}, H_{+}\right.$, $\left.H_{-}, H_{-}\right\}, \operatorname{diag} \hat{E}=\{E, E, E, E\}$.

\section{B. $S U(2)$ and $\mathcal{C P} \mathcal{T}_{ \pm}$symmetry}

Let us now analyze the model $\hat{\mathcal{I}}_{s u 2}$ in more detail. First we verify the $S U(2)$ symmetry of the action and its effect on the different types of fields. Noting that the change in the complex scalar fields is $\delta \phi_{j}^{k}=i \alpha_{a} T_{a}^{k l} \phi_{j}^{l}$, with the generators $T_{a}$ of the 
symmetry transformation taken to be standard Pauli matrices $\sigma_{a}, a=1,2,3$, we directly identify the infinitesimal changes for the real component fields as

$$
\begin{array}{ll}
\delta \varphi_{j}^{1}=-\alpha_{1} \chi_{j}^{2}+\alpha_{2} \varphi_{j}^{2}-\alpha_{3} \chi_{j}^{1}, & \delta \chi_{j}^{1}=\alpha_{1} \varphi_{j}^{2}+\alpha_{2} \chi_{j}^{2}+\alpha_{3} \varphi_{j}^{1}, \\
\delta \varphi_{j}^{2}=-\alpha_{1} \chi_{j}^{1}-\alpha_{2} \varphi_{j}^{1}+\alpha_{3} \chi_{j}^{2}, & \delta \chi_{j}^{2}=\alpha_{1} \varphi_{j}^{1}-\alpha_{2} \chi_{j}^{1}-\alpha_{3} \varphi_{j}^{2} .
\end{array}
$$

It is easily verified that the Hermitian action $\hat{\mathcal{I}}_{s u 2}$ remains invariant under the transformations (3.10), (3.11). For the 4- and 8-component fields the symmetries (3.10), (3.11) then translate into

$$
\begin{gathered}
\delta \Phi=-\alpha_{1}\left(\sigma_{1} \otimes \sigma_{3}\right) \Psi+i \alpha_{2}\left(\sigma_{2} \otimes \mathbb{I}\right) \Phi-\alpha_{3}\left(\sigma_{3} \otimes \sigma_{3}\right) \Psi \\
\delta \Psi=\alpha_{1}\left(\sigma_{1} \otimes \sigma_{3}\right) \Phi+i \alpha_{2}\left(\sigma_{2} \otimes \mathbb{I}\right) \Psi+\alpha_{3}\left(\sigma_{3} \otimes \sigma_{3}\right) \Phi \\
\delta F=i\left[-\alpha_{1}\left(\sigma_{2} \otimes \sigma_{1} \otimes \sigma_{3}\right)+\alpha_{2}\left(\mathbb{I} \otimes \sigma_{2} \otimes \mathbb{I}\right)-\alpha_{3}\left(\sigma_{2} \otimes \sigma_{3} \otimes \sigma_{3}\right)\right] F
\end{gathered}
$$

with $\otimes$ denoting the standard tensor product. These expressions may be applied to the action in the forms (3.7) and (3.8), respectively, to verify the $S U(2)$ symmetry.

The antilinear $\mathcal{C P} \mathcal{T}_{ \pm}$-symmetries manifest themselves as

$$
\begin{aligned}
\mathcal{C P} \mathcal{T}_{ \pm}: & \varphi_{j}^{k}\left(x_{\mu}\right) \rightarrow \mp(-1)^{j} \varphi_{j}^{k}\left(-x_{\mu}\right), \\
& \chi_{j}^{k}\left(x_{\mu}\right) \rightarrow \pm(-1)^{j} \chi_{j}^{k}\left(-x_{\mu}\right), \\
\Phi\left(x_{\mu}\right) \rightarrow & \pm \Phi\left(-x_{\mu}\right), \quad \Psi\left(x_{\mu}\right) \rightarrow \mp \Psi\left(-x_{\mu}\right), \\
& F\left(x_{\mu}\right) \rightarrow \pm\left(\sigma_{3} \otimes \mathbb{I} \otimes \mathbb{I}\right) F\left(-x_{\mu}\right),
\end{aligned}
$$

which can be verified in (3.6), (3.7) and (3.8), respectively.

\section{C. $S U(2)$-symmetry invariant and breaking vacua}

Let us now compute the vacua from (2.4) with potential as specified in (3.6). We find there are only two types of vacua, that either break or respect the $S U(2)$ symmetry,

$$
\begin{gathered}
F_{0}^{b}=(x,-a x, y,-a y, z, a z, \pm R, \pm a R), \\
F_{0}^{s}=(0,0,0,0,0,0,0,0),
\end{gathered}
$$

respectively. We introduced the notation $x:=\varphi_{1}^{0,1}, y:=\varphi_{1}^{0,2}$, $z:=\chi_{1}^{0,1}$, for the vacuum field components and $a:=\mu^{2} / m_{2}^{2}$, $R:=\sqrt{r^{2}-\left(x^{2}+y^{2}+z^{2}\right)}, r:=4\left(\mu^{2}+m_{1}^{2} m_{2}^{2}\right) / g m_{2}^{2}$ for convenience. We note that the defining relation for $R$ can be interpreted as a three sphere in $\mathbb{R}^{4}$ with center $(0,0,0,0)$ and radius $r$, which is the geometrical configuration expected from its topological isomorphism with the $S U(2)$-group manifold. We note that the points $\mu^{2}=-m_{1}^{2} m_{2}^{2}$ are special as there the three sphere collapses to a point and the symmetry of the vacuum is restored $F_{0}^{b} \rightarrow F_{0}^{s}$.

The symmetry properties of the vacua are easily established. Identifying the generators $T_{a}$ of the symmetry transformation as Pauli matrices, where we drop the usual factor of $1 / 2$, we compute the action on the vacuum states, say $\phi_{j}^{0}=\left(\phi_{j}^{0,1}, \phi_{j}^{0,2}\right)^{T}$ for $j=1,2$. We find

$$
\begin{aligned}
& T_{1} \phi_{j}^{0}=\left(\phi_{j}^{0,2}, \phi_{j}^{0,1}\right)^{T}, \quad T_{2} \phi_{j}^{0}=\left(-i \phi_{j}^{0,2}, i \phi_{j}^{0,1}\right)^{T}, \\
& T_{3} \phi_{j}^{0}=\left(\phi_{j}^{0,1},-\phi_{j}^{0,2}\right)^{T},
\end{aligned}
$$

so that for nonzero fields the vacuum will always break the symmetry with respect to the action of $T_{1}$ and $T_{2}$. The action of $T_{3}$ seems to require only $\phi_{j}^{0,2}=0$, in order to achieve invariance. However, apart from $F_{0}^{s}$ there is no possible choice for the fields in $F_{0}^{b}$ so that $\phi_{j}^{0,1} \neq 0$ in that case.

Let us now make use of the argument in (2.8) and employ the $S U(2)$ symmetry to transform the vacuum $F_{0}^{b}$ into a physically equivalent but more manageable one. Choosing two simple target vacua $\breve{\phi}_{1}^{0}$ and $\breve{\phi}_{2}^{0}$, we attempt therefore to simultaneously solve the two equations

$$
e^{i \alpha_{a} T_{a}} \phi_{1}^{0}=[\cos \rho \mathbb{I}+i \sin \rho(\mathbf{n} \cdot \boldsymbol{\sigma})] \phi_{1}^{0}=\check{\phi}_{1}^{0}=\left(\begin{array}{l}
0 \\
\pm i r
\end{array}\right),
$$

$$
e^{i \alpha_{a} T_{a}} \phi_{2}^{0}=[\cos \rho \mathbb{I}+i \sin \rho(\mathbf{n} \cdot \boldsymbol{\sigma})] \phi_{2}^{0}=\check{\phi}_{2}^{0}=\left(\begin{array}{l}
0 \\
\pm a r
\end{array}\right),
$$

by using the well-known formula $e^{i \rho \mathbf{n} \cdot \boldsymbol{\sigma}}=\cos \rho \mathbb{I}+$ $i \cos \rho(\mathbf{n} \cdot \boldsymbol{\sigma})$ with $\rho=\sqrt{\alpha_{1}^{2}+\alpha_{2}^{2}+\alpha_{3}^{2}}, \mathbf{n}=\left(\alpha_{1}, \alpha_{2}, \alpha_{3}\right) / \rho$ and $T_{a}=\sigma_{a}$. The vacuum fields are parametrized as

$$
\begin{aligned}
& \phi_{1}^{0}=\left(\begin{array}{c}
\varphi_{1}^{0,1}+i \chi_{1}^{0,1} \\
\varphi_{1}^{0,2}+i \chi_{1}^{0,2}
\end{array}\right)=\left(\begin{array}{c}
x+i z \\
y+i R
\end{array}\right), \quad \text { and } \\
& \phi_{2}^{0}=\left(\begin{array}{c}
\varphi_{2}^{0,1}+i \chi_{2}^{0,1} \\
\varphi_{2}^{0,2}+i \chi_{2}^{0,2}
\end{array}\right)=\left(\begin{array}{c}
-a z+i a x \\
-a R+i a y
\end{array}\right),
\end{aligned}
$$


so that the form of the target vacuum is motivated by setting $x=y=z=0$. We only keep one of the sign in (3.18) and solve (3.21), (3.22) by

$x=\frac{r}{\rho} \sin \rho \alpha_{1}, \quad y=-\frac{r}{\rho} \sin \rho \alpha_{3}, \quad z=-\frac{r}{\rho} \sin \rho \alpha_{2}$,

so that $R=r \cos \rho$. For the vacuum $F_{0}^{b}$ this translates with (3.14) into

$$
\mathcal{T} F_{0}^{b}=\check{F}_{0}^{b},
$$

where

$$
\begin{gathered}
\mathcal{T}=\cos (\rho) \mathbb{I}_{8}-i \frac{\sin (\rho)}{\rho}\left[\alpha_{1}\left(\sigma_{2} \otimes \sigma_{1} \otimes \sigma_{3}\right)\right. \\
\left.-\alpha_{2}\left(\mathbb{I} \otimes \sigma_{2} \otimes \mathbb{I}\right)+\alpha_{3}\left(\sigma_{2} \otimes \sigma_{3} \otimes \sigma_{3}\right)\right], \\
\check{F}_{0}^{b}=(0,0,0,0,0,0, \pm r, \pm a r) .
\end{gathered}
$$

We note that $\operatorname{det} \mathcal{T}=1$ and as required $\mathcal{T}^{T}=\mathcal{T}^{-1}$. Evidently $\breve{F}_{0}^{b}$ is of a more convenient form of the vacuum than $F_{0}^{b}$ and we shall therefore use it from here on.

\section{Mass squared matrices and null vectors}

Next we use the different vacua and expand the potentials around them to determine the mass squared matrix according to the definition in (2.7). Expanding first around the $S U(2)$-symmetric vacuum $F_{0}^{s}$ we find the mass squared matrix

$$
M_{s}^{2}=\left(\begin{array}{cccccccc}
-m_{1}^{2} & \mu^{2} & 0 & 0 & 0 & 0 & 0 & 0 \\
-\mu^{2} & -m_{2}^{2} & 0 & 0 & 0 & 0 & 0 & 0 \\
0 & 0 & -m_{1}^{2} & \mu^{2} & 0 & 0 & 0 & 0 \\
0 & 0 & -\mu^{2} & -m_{2}^{2} & 0 & 0 & 0 & 0 \\
0 & 0 & 0 & 0 & -m_{1}^{2} & -\mu^{2} & 0 & 0 \\
0 & 0 & 0 & 0 & \mu^{2} & -m_{2}^{2} & 0 & 0 \\
0 & 0 & 0 & 0 & 0 & 0 & -m_{1}^{2} & -\mu^{2} \\
0 & 0 & 0 & 0 & 0 & 0 & \mu^{2} & -m_{2}^{2}
\end{array}\right) \text {, }
$$

with two fourfold degenerate eigenvalues

$$
\lambda_{ \pm}^{s}=-\frac{1}{2}\left(m_{1}^{2}+m_{2}^{2} \pm \sqrt{\left(m_{1}^{2}-m_{2}^{2}\right)^{2}-4 \mu^{4}}\right) .
$$

As expected from (2.7) there are no Goldstone bosons emerging in this $S U(2)$-invariant case.

Expanding instead around the $S U(2)$-symmetry breaking vacuum $F_{0}^{b}$, we obtain the mass squared matrix

$$
M_{b}^{2}=\left(\begin{array}{cccccccc}
\frac{g\left(\varphi_{1}^{1}\right)^{2}}{2}+\frac{\mu^{4}}{m_{2}^{2}} & \mu^{2} & \frac{g \varphi_{1}^{1} \varphi_{1}^{2}}{2} & 0 & \frac{g \varphi_{1}^{1} \chi_{1}^{1}}{2} & 0 & -\frac{\varphi_{1}^{1} g R}{2} & 0 \\
-\mu^{2} & -m_{2}^{2} & 0 & 0 & 0 & 0 & 0 & 0 \\
\frac{g \varphi_{1}^{1} \varphi_{1}^{2}}{2} & 0 & \frac{g\left(\varphi_{1}^{2}\right)^{2}}{2}+\frac{\mu^{4}}{m_{2}^{2}} & \mu^{2} & \frac{g \varphi_{1}^{2} \chi_{1}^{1}}{2} & 0 & -\frac{\varphi_{1}^{2} g R}{2} & 0 \\
0 & 0 & -\mu^{2} & -m_{2}^{2} & 0 & 0 & 0 & 0 \\
\frac{g \varphi_{1}^{1} \chi_{1}^{1}}{2} & 0 & \frac{g \varphi_{1}^{2} \chi_{1}^{1}}{2} & 0 & \frac{g\left(\varphi_{1}^{2}\right)^{2}}{2}+\frac{\mu^{4}}{m_{2}^{2}} & -\mu^{2} & -\frac{\chi_{1}^{1} g R}{2} & 0 \\
0 & 0 & 0 & 0 & \mu^{2} & -m_{2}^{2} & 0 & 0 \\
-\frac{\varphi_{1}^{1} g R}{2} & 0 & -\frac{\varphi_{1}^{2} g R}{2} & 0 & -\frac{\chi_{1}^{1} g R}{2} & 0 & \frac{g^{2} R^{2}}{2}+\frac{\mu^{4}}{m_{2}^{2}} & -\mu^{2} \\
0 & 0 & 0 & 0 & 0 & 0 & \mu^{2} & -m_{2}^{2}
\end{array}\right) .
$$

For convenience we defined here $K:=3 \mu^{4} / 2 m_{2}^{2}+m_{1}^{2}-$ $m_{2}^{2} / 2$ and $L:=\mu^{4}+m_{1}^{2} m_{2}^{2}$. We confirm the expectation from Goldstone's theorem to find three massless Goldstone bosons in the symmetry breaking sector, since none of the three $S U(2)$ generators leaves the vacuum $F_{0}^{b}$ invariant.

According to the relation (2.7) we may compute the corresponding null vectors directly from the $S U(2)$ symmetry transformation. When applying the infinitesimal 
Physical region

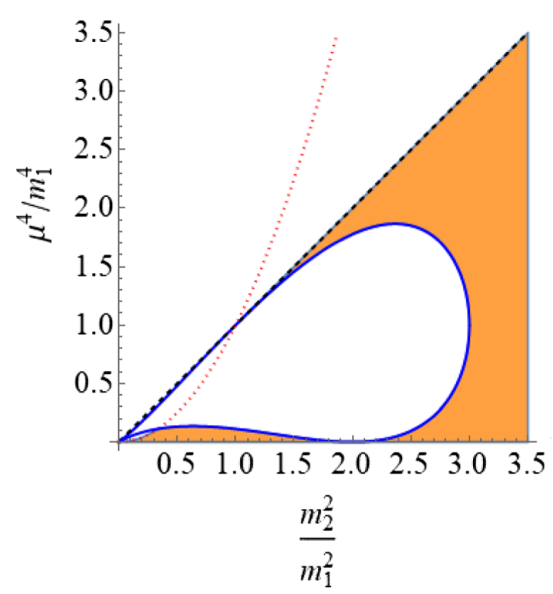

Physical region

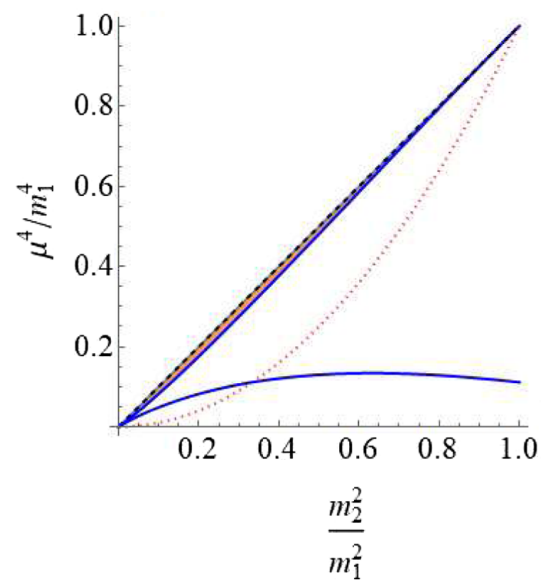

FIG. 1. Physical regions in parameter space bounded by exceptional and zero exceptional points as a function of $\left(\mu^{4} / m_{1}^{4}, m_{2}^{2} / m_{1}^{2}\right)$ for the theory expanded around the $S U(2)$-symmetry breaking vacuum. Left panel for $c_{1}=-c_{2}=1$ and right panel for $c_{1}=-c_{2}=-1$.

changes for the component fields (3.10) and (3.11) to the vacuum $F_{0}^{b}$, we obtain the vectors

$\nu_{1}^{0}=\frac{1}{\sqrt{N}}\left\{R,-a R,-\chi_{1}^{1}, \frac{\mu^{2} \chi_{1}^{1}}{m_{2}^{2}}, \varphi_{1}^{2}, \frac{\mu^{2} \varphi_{1}^{2}}{m_{2}^{2}}, \varphi_{1}^{1}, \frac{\mu^{2} \varphi_{1}^{1}}{m_{2}^{2}}\right\}$

$\nu_{2}^{0}=\frac{1}{\sqrt{N}}\left\{\varphi_{1}^{2},-\frac{\mu^{2} \varphi_{1}^{2}}{m_{2}^{2}},-\varphi_{1}^{1}, \frac{\mu^{2} \varphi_{1}^{1}}{m_{2}^{2}},-R,-a R,-\chi_{1}^{1},-\frac{\mu^{2} \chi_{1}^{1}}{m_{2}^{2}}\right\}$,

$\nu_{3}^{0}=\frac{1}{\sqrt{N}}\left\{-\chi_{1}^{1}, \frac{\mu^{2} \chi_{1}^{1}}{m_{2}^{2}},-R, a R, \varphi_{1}^{1}, \frac{\mu^{2} \varphi_{1}^{1}}{m_{2}^{2}},-\varphi_{1}^{2},-\frac{\mu^{2} \varphi_{1}^{2}}{m_{2}^{2}}\right\}$,

with $N:=-4 L \lambda_{4,5,6}^{b} / g m_{2}^{4}$. These vectors have been normalized with regard to the $\mathcal{C P} \mathcal{T}$-inner product $\langle x \mid I y\rangle$. We verify that the $\nu_{i}^{0}, i=1,2,3$, are indeed null vectors of $M_{b}^{2}$. Furthermore, we observe from the normalization constant that at the zero exceptional points, i.e., for $\mu^{4}=m_{2}^{4}$ when $\lambda_{4,5,6}^{b}=0$ and $\mu^{4}=-m_{1}^{2} m_{2}^{2}$ when $\lambda_{-}^{b}=0$, these vectors are not defined. We may ignore the case $\lambda_{-}^{b}=0$ in what follows as in this case the $S U(2)$ symmetry is restored with $\breve{F}_{0}^{b} \rightarrow F_{0}^{s}$.

\section{E. Physical regions}

We will now analyze the parameter space of the system and identify the physical regions based on a meaningful mass squared matrix. To cover all possible cases we are setting therefore in all expressions $m_{i}^{2} \rightarrow c_{i} m_{i}^{2}$. For the model expanded around the broken vacuum the physical regions are then determined by $\lambda_{ \pm}^{b} \geq 0, \lambda_{4,5,6}^{b} \geq 0$ corresponding to the four inequalities
$K \geq 0, \quad L \leq 0, \quad K^{2}+2 L \geq 0, \quad c_{2} \mu^{4} \geq c_{2} m_{2}^{4}$,

for the four cases $c_{1}= \pm 1, c_{2}= \pm 1$. All constraints can be expressed as functions of the two ratios $\left(\mu^{4} / m_{1}^{4}, m_{2}^{2} / m_{1}^{2}\right)$. We find that no solutions exists for $c_{1}=c_{2}$, apart from setting $\mu=m_{2}=0$, so that in these two case the model is unphysical. The physical regions for the remaining two cases $c_{1}=-c_{2}= \pm 1$ are depicted in Fig. 1 .

The two different cases depicted in Fig. 1 do not have any physical regions that intersect. The case $c_{1}=-c_{2}=1$ was also analyzed within the surface term approach in [20] and our results appear to match exactly. The case $c_{1}=$ $-c_{2}=-1$ was not dealt with in [20], but as depicted in Fig. 1, it also contains a well-defined small physical region. We note that for our model with two complex scalar fields the physical regions have no boundary corresponding to singularities, which appears to be a feature only occurring for the theories with more complex scalar fields, see [19].

Finally in Fig. 2 we also depict the physical regions for the model expanded around the $S U(2)$-invariant vacuum.

Here only the case $c_{1}=c_{2}=1$ does not contain a physical region apart from $\mu=m_{2}=0$. The three different cases depicted in Fig. 2 do not have any physical regions that intersect, apart from the small region near the origin. Comparing Figs. 1 and 2 we also notice that cases with equal choices for the $c_{i}$ do not share physical regions. This implies that for any particular physical model the breaking of the $S U(2)$ symmetry leads to an unphysical model and in reverse also that some unphysical models become physical when the $S U(2)$ symmetry is broken.

\section{F. The Goldstone bosons in the $\mathcal{P} \mathcal{T}$-symmetric regime}

We may now compute the Goldstone bosons in terms of the original fields in a similar fashion as discussed in [19]. 


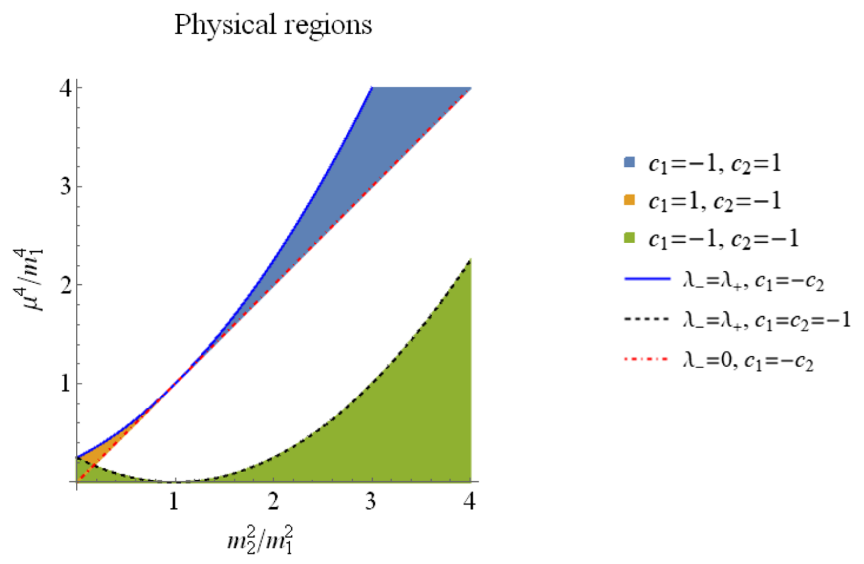

FIG. 2. Physical regions in parameter space bounded by exceptional and zero exceptional points as function of $\left(\mu^{4} / m_{1}^{4}\right.$, $m_{2}^{2} / m_{1}^{2}$ ) for the theory expanded around the $S U(2)$-symmetry invariant vacuum.

Defining for this purpose the remaining right eigenvectors $v_{i}, i=4, \ldots, 8$, and a matrix $U$ containing all of them as column vectors as

$$
\begin{aligned}
M_{b}^{2} v_{i} & =\lambda_{i}^{b} v_{i}, \quad U:=\left(v_{1}, v_{4}, v_{2}, v_{5}, v_{3}, v_{6}, v_{-}, v_{+}\right) \\
i & =1, \ldots, 6, \pm
\end{aligned}
$$

we diagonalize the mass squared matrix by means of the similarity transformation $U^{-1} M_{b}^{2} U=D$ with $\operatorname{diag} D=$ $\left(\lambda_{1}^{b}, \lambda_{4}^{b}, \lambda_{2}^{b}, \lambda_{5}^{b}, \lambda_{3}^{b}, \lambda_{6}^{b}, \lambda_{-}^{b}, \lambda_{+}^{b}\right)=\left(m_{1}^{2}, \ldots, m_{8}^{2}\right)$. For $\mu^{4} \neq m_{2}^{4}$ and $K^{2} \neq-2 L$, that are the zero and standard exceptional points, we define the fields $\psi_{i}$ with masses $m_{i}$ by rewriting the squared mass term as

$F^{T} M_{b}^{2} F=\sum_{k=1}^{8} m_{k}^{2} \psi_{k}^{2}=\sum_{k=1}^{8} m_{k}^{2}\left(F^{T} I U\right)_{k}\left(U^{-1} F\right)_{k}$

Hence, the three Goldstone fields are identified as

$$
\psi_{\ell}^{\mathrm{Gb}}:=\sqrt{\left(F^{T} I U\right)_{\ell}\left(U^{-1} F\right)_{\ell}}, \quad \ell=1,3,5 .
$$

Setting in $M_{b}^{2}$ the fields $\chi_{1}^{0,1}, \varphi_{1}^{0,1}, \varphi_{1}^{0,2}$ to zero we compute

$$
U=\left(\begin{array}{ccccc}
H_{-} & 0 & 0 & 0 & 0 \\
0 & H_{-} & 0 & 0 & 0 \\
0 & 0 & H_{+} & 0 & 0 \\
0 & 0 & 0 & \lambda_{-}^{b}+m_{2}^{2} & \lambda_{+}^{b}+m_{2}^{2} \\
0 & 0 & 0 & \mu^{2} & \mu^{2}
\end{array}\right),
$$

with $\operatorname{det} U=2 \mu^{2}\left(\mu^{4}-m_{2}^{4}\right)^{3} \sqrt{K^{2}+2 L}$, so that the explicit form of the Goldstone boson fields in the original fields result in

$$
\begin{aligned}
& \psi_{1}^{\mathrm{Gb}}=\frac{\mu^{2} \varphi_{2}^{1}-m_{2}^{2} \chi_{1}^{1}}{\sqrt{m_{2}^{4}-\mu^{4}}}, \quad \psi_{3}^{\mathrm{Gb}}=\frac{m_{2}^{2} \varphi_{1}^{2}+\mu^{2} \chi_{2}^{2}}{\sqrt{m_{2}^{4}-\mu^{4}}}, \\
& \psi_{5}^{\mathrm{Gb}}=\frac{m_{2}^{2} \varphi_{1}^{1}+\mu^{2} \chi_{2}^{1}}{\sqrt{m_{2}^{4}-\mu^{4}}} .
\end{aligned}
$$

As $U$ is not invertible at the exceptional points for $\mu^{4}=m_{2}^{4}$ and $K^{2}=-2 L$, we need to treat these cases separately. We note that these expressions differ from those obtained in [20].

\section{G. The Goldstone bosons at the exceptional point}

At the standard exceptional point, i.e., when $K^{2}=-2 L$ and hence $\lambda_{+}^{b}=\lambda_{-}^{b}$, the two eigenvectors $v_{-}$and $v_{+}$ coalesce so that the matrix $U$ is no longer invertible and the Goldstone boson fields may take on a different form as found in [19]. Instead of diagonalizing the mass squared matrix we can convert it into Jordan normal form by means of a similarity transformation. Making $m_{1}$ the dependent variable, the exceptional point occurs when $m_{1}^{2}= \pm \mu^{2}-$ $m_{2}^{2} / 2-3 \mu^{4} / 2 m_{2}^{2}$ so that the Jordan normal form becomes

$$
\begin{aligned}
\operatorname{diag} D_{e} & =\left(0, \lambda_{e}^{b}, 0, \lambda_{e}^{b}, 0, \lambda_{e}^{b}, \Lambda\right), \quad \lambda_{e}^{b}=\frac{\mu^{4}}{m_{2}^{2}}-m_{2}^{2} \\
\Lambda & =\left(\begin{array}{ll} 
\pm \mu^{2}-m_{2}^{2} & \pm(\alpha-\beta) \mu^{2} \\
0 & \pm \mu^{2}-m_{2}^{2}
\end{array}\right)
\end{aligned}
$$

which can be obtained from the similarity transformation $U_{e}^{-1} M_{e}^{2} U_{e}=D_{e}$ with $U_{e}$ equaling $U$ with the lower right block replaced by

$$
\left(\begin{array}{ll}
1 & \alpha \\
1 & \beta
\end{array}\right)
$$

We compute now det $U=(\alpha-\beta)\left(\mu^{4}-m_{2}^{4}\right)^{3}$. Defining the Goldstone boson fields by the same formal expression as in (3.38), but with $U$ replaced by $U_{e}$, we obtain at the exceptional point the same expressions as in (3.38). It is worth noting that the two degenerate fields take on the form

$$
\psi_{+, e}=\psi_{-, e}=\frac{\sqrt{\left(\varphi_{2}^{2}-\chi_{1}^{2}\right)\left(\alpha \varphi_{2}^{2}-\beta \chi_{1}^{2}\right)}}{\sqrt{\beta-\alpha}} .
$$

We note that it is by far not obvious that the Goldstone boson fields acquire the same form in the $\mathcal{P} \mathcal{T}$-symmetric regime as at the exceptional point. This is more a coincidence due to the special nature of the mass matrix rather than a general feature. When considering models with more than two scalar fields this no longer holds even for the Abelian case as observed in [19]. In [20] this regime was not analyzed separately.

The behavior at the zero exceptional points is similar as discussed in more detail in [19]. For $\mu^{4}=m_{2}^{4}$ when 
$\lambda_{4,5,6}^{b}=0$ we cannot find a matrix $U$ that diagonalizes $M^{2}$ so that the Goldstone bosons are expressible in terms of the original fields in the action. The zero exceptional point for $\mu^{4}=-m_{1}^{2} m_{2}^{2}$ when $\lambda_{-}^{b}=0$ needs no discussion as at this point the original $S U(2)$ symmetry is restored.

\section{CONCLUSIONS AND OUTLOOK}

Using a pseudo-Hermitian approach to treat non-Hermitian quantum field theories we found that the Goldstone theorem also holds when the global symmetry group is non-Abelian. The explicit form for the Goldstone boson in the $\mathcal{P} \mathcal{T}$ symmetric regime and at the standard exceptional points can be found explicitly, although using different diagonalization procedures for the mass squared matrix. At the zero exceptional point the Goldstone boson cannot be identified. When the analysis of our model overlaps with the one carried out in [20] employing the surface term approach, the physical regions coincide exactly. However, the explicit forms of the Goldstone bosons are different.

There are some obvious further extensions to this investigation, that would be interesting to carry out, such as the treatment of models with different Lie symmetry groups and the augmentation of the amount of complex scalar fields. Most interesting, with regard to the comparison with the surface term approach, is the investigation of the Higgs mechanism within the presented framework as that aspect will produce more features and predictions that are clearly distinct in the two approaches [29].

Furthermore, it would be very interesting to establish a closer link between studies carried on non-Hermitian systems in $1+1$ dimensions. In principle, the Goldstone theorem does not apply for dimension $d \leq 2$ as in those settings the breaking of continuous symmetries inevitably leads to infrared divergent correlation functions. However, in [30] it was argued that the Mermin-Wagner theorem no longer applies for the continuous $S O(N)$ symmetry with $N<2$ as it cannot be realized as unitary operations on vector fields. This feature was exploited in [30] to identify a Goldstone phase for a non-Hermitian system.

\section{ACKNOWLEDGMENTS}

We would like to thank Peter Millington for interesting discussions and Hubert Saleur for pointing out Ref. [30] to us.
[1] C. M. Bender, V. Branchina, and E. Messina, Critical behavior of the P T-symmetric $i \phi^{3}$ quantum field theory, Phys. Rev. D 87, 085029 (2013).

[2] A. M. Shalaby, Vacuum structure and PT-symmetry breaking of the non-Hermetian $i \phi^{3}$ theory, Phys. Rev. D 96, 025015 (2017).

[3] C. M. Bender, N. Hassanpour, S. Klevansky, and S. Sarkar, PT-symmetric quantum field theory in D dimensions, Phys. Rev. D 98, 125003 (2018).

[4] J. Alexandre, C. M. Bender, and P. Millington, NonHermitian extension of gauge theories and implications for neutrino physics, J. High Energy Phys. 11 (2015) 111.

[5] V. E. Rochev, Hermitian vs PT-symmetric scalar Yukawa model, J. Mod. Phys. 7, 899 (2016).

[6] A. Y. Korchin and V. A. Kovalchuk, Decay of the Higgs boson to $\tau^{-} \tau^{+}$and non-Hermiticy of the Yukawa interaction, Phys. Rev. D 94, 076003 (2016).

[7] L. Gouba, The Yukawa model in one space-one time simensions, in Mathematical Structures and Applications (Springer, New York, 2018), pp. 225-233.

[8] C. M. Bender, H. F. Jones, and R. J. Rivers, Dual PTsymmetric quantum field theories, Phys. Lett. B 625, 333 (2005).

[9] C. M. Bender and K. A. Milton, A nonunitary version of massless quantum electrodynamics possessing a critical point, J. Phys. A 32, L87 (1999).

[10] K. A. Milton, E. K. Abalo, P. Parashar, N. Pourtolami, and J. Wagner, PT-symmetric quantum electrodynamics and unitarity, Phil. Trans. R. Soc. A 371, 20120057 (2013).
[11] C. M. Bender, N. Hassanpour, S. P. Klevansky, and S. Sarkar, PT-symmetric quantum field theory in D dimensions, Phys. Rev. D 98, 125003 (2018).

[12] Y. Nambu and G. Jona-Lasinio, Dynamical model of elementary particles based on an analogy with superconductivity. II, Phys. Rev. 124, 246 (1961).

[13] J. Goldstone, Field theories with superconductor solutions, Nuovo Cimento (1955-1965) 19, 154 (1961).

[14] F. Englert and R. Brout, Broken Symmetry and the Mass of Gauge Vector Mesons, Phys. Rev. Lett. 13, 321 (1964).

[15] P. W. Higgs, Broken Symmetries and the Masses of Gauge Bosons, Phys. Rev. Lett. 13, 508 (1964).

[16] G. S. Guralnik, C. R. Hagen, and T. W. B. Kibble, Global Conservation Laws and Massless Particles, Phys. Rev. Lett. 13, 585 (1964).

[17] J. Alexandre, J. Ellis, P. Millington, and D. Seynaeve, Spontaneous symmetry breaking and the Goldstone theorem in non-Hermitian field theories, Phys. Rev. D 98, 045001 (2018).

[18] P. D. Mannheim, Goldstone bosons and the Englert-BroutHiggs mechanism in non-Hermitian theories, Phys. Rev. D 99, 045006 (2019).

[19] A. Fring and T. Taira, Goldstone bosons in different PT-regimes of non-Hermitian scalar quantum field theories, Nucl. Phys. B950, 114834 (2019).

[20] J. Alexandre, J. Ellis, P. Millington, and D. Seynaeve, Spontaneously breaking non-Abelian gauge symmetry in non-Hermitian field theories, Phys. Rev. D 101, 035008 (2020). 
[21] E. Wigner, Normal form of antiunitary operators, J. Math. Phys. (N.Y.) 1, 409 (1960).

[22] J. Alexandre and C. M. Bender, Foldy-Wouthuysen transformation for non-Hermitian Hamiltonians, J. Phys. A 48, 185403 (2015).

[23] J. Alexandre, P. Millington, and D. Seynaeve, Symmetries and conservation laws in non-Hermitian field theories, Phys. Rev. D 96, 065027 (2017).

[24] H. B. Geyer, F. G. Scholtz, and I. Snyman, QuasiHermiticity and the role of a metric in some boson Hamiltonians, Czech. J. Phys. 54, 1069 (2004).

[25] C. M. Bender and S. Boettcher, Real Spectra in NonHermitian Hamiltonians Having PT Symmetry, Phys. Rev. Lett. 80, 5243 (1998).
[26] A. Mostafazadeh, Pseudo-Hermitian representation of quantum mechanics, Int. J. Geom. Methods Mod. Phys. 07, 1191 (2010).

[27] C. M. Bender, P. E. Dorey, C. Dunning, A. Fring, D. W. Hook, H. F. Jones, S. Kuzhel, G. Levai, and R. Tateo, PT Symmetry: In Quantum and Classical Physics (World Scientific, Singapore, 2019).

[28] F. J. Dyson, Thermodynamic behavior of an ideal Ferromagnet, Phys. Rev. 102, 1230 (1956).

[29] A. Fring and T. Taira (to be published).

[30] J.-L. Jacobsen, N. Read, and H. Saleur, Dense Loops, Supersymmetry, and Goldstone Phases in Two Dimensions, Phys. Rev. Lett. 90, 090601 (2003). 